\title{
EVALUACIÓN DEL RENDIMIENTO DEL ARIETE HIDRÁULICO BAH- 1.1/2 VARIANDO LA LONGITUD DE LA TUBERÍA DE ALIMENTACIÓN Y CONDICIONES DE OPERACIÓN DE LA VÁLVULA DE IMPULSO
}

\author{
PERFORMANCE EVALUATION OF THE HYDRAULIC RAM BAH-1.1/2 VARYING THE \\ LENGTH OF THE FEED PIPE AND OPERATING CONDITIONS OF THE IMPULSE VALVE
}

\author{
${ }^{1}$ José Bernardino Arapa Quispe
}

\begin{abstract}
Resumen
Una bomba de ariete hidráulico es un dispositivo, que usa la energía de una caída de agua, para generar potencia y poder elevar un porcentaje del agua de ingreso a una altura mayor que la altura de caída, de forma continua y automática sin ninguna otra energía externa.El objetivo del presente trabajo es evaluar el rendimiento del ariete hidráulico modelo BAH 1.1/2, utilizando tubería de alimentación de PVC de 1.1/2" de diámetro y variando las longitudes en 1.0, 2.0 y $3.0 \mathrm{~m}$, para diferentes condiciones de operación de la válvula de impulso, variando en 3, 6 y $9 \mathrm{~mm}$ la luz de la carreras de cierre. La evaluación del ariete hidráulico fue realizada en el Laboratorio de Hidráulica y Mecánica de Fluidos del Departamento de Recursos Hídricos de la Facultad de Ingeniería Agrícola de la Universidad Nacional Agraria La Molina. Siendo el banco de prueba utilizado una estructura de material noble de 5.18 x 1.14 x $0.80 \mathrm{~m}$ de largo, ancho y alto respectivamente, abastecido de agua con una electrobomba de $0.85 \mathrm{HP}(0.60 \mathrm{~kW})$ de $10-80 \mathrm{l} / \mathrm{min}$ de caudal. La bomba de ariete evaluada fue ensamblada principalmente con accesorios comerciales de fierro galvanizado, acero inoxidable y bronce. Ensayo realizado con altura de $1.0 \mathrm{~m}$ de carga de agua, alimentada con tubería de PVC de $40 \mathrm{~mm}$ $(1.1 / 2$ ") y tubería de descarga de FG de $21 \mathrm{~mm}$ (1/2"), con $62 \mathrm{~mm}$ de diámetro de paso de válvula impulso del ariete y 1500 gramos de peso en el resorte del vástago de la válvula de cierre. Los resultados permiten concluir que la bomba de ariete BAH 1.1/2 evaluada opera regularmente en las diferentes longitudes y carreras evaluadas, logrando mejor eficiencia con tubería de $3 \mathrm{~m}$ de longitud y con carreras de la válvula de impulso de $9 \mathrm{~mm}$, con lo cual se logra alcanzar alturas de bombeo de 10, 20, 25 y 30 m con eficiencia $64.16,60.34$ y $59.11 \%$ con caudales bombeados de $1.26,0.99$ y $0.80 \mathrm{l} / \mathrm{min}$, consiguiendo bombear un volumen por día de $1814.40,1425.60$ y 1152 litros.
\end{abstract}

Palabras Claves: Ariete Hidráulico, Bomba de Ariete, Energía Renovable, Energía Limpia, Válvula de Impulso.

\begin{abstract}
A hydraulic ram pump is a device that uses the energy of falling water to generate power and to raise a percentage of incoming water to a greater height than the height of fall continuously and automatically without any other external energy. The aim of this study is to evaluate the performance of the hydraulic ram model BAH $1.1 / 2$, using PVC pipe feeding 1.1 / 2 "diameter and varying lengths in 1.0, 2.0 and $3.0 \mathrm{~m}$, for different operating conditions of the impulse valve, varying in 3,6 and $9 \mathrm{~mm}$ light closure races. The evaluation of the hydraulic ram was conducted at the Laboratory of Hydraulics and Fluid Mechanics Department of Water Resources of the Faculty of Agricultural Engineering of the Universidad Nacional Agraria La Molina. As the test bench used a noble material structure of $5.18 \times 1.14 \times 0.80 \mathrm{~m}$ in length, width and height respectively supplied water with an electric pump $0.85 \mathrm{HP}(0.60 \mathrm{~kW})$ of $10-801 / \mathrm{min}$ flow. The tested ram pump was assembled primarily with commercial accessories of galvanized iron, stainless steel and bronze. Test at height of $1.0 \mathrm{~m}$ of head of water, supplied with PVC pipe $40 \mathrm{~mm}(1.1 / 2$ " $)$ and FG discharge pipe of $21 \mathrm{~mm}$ $(1 / 2$ "), with $62 \mathrm{~mm}$ gate valve size, impulse ram and 1500 grams in the spring valve stem sealing. The results suggest that the tested ram pump BAH 1.1 / 2 operates regularly in different lengths and tested races, achieving better efficiency with pipe $3 \mathrm{~m}$ in length and with impulse valve of $9 \mathrm{~mm}$, which achieved pumping heights of 10, 20, 25 and $30 \mathrm{~m}$ with efficiency $64.16,60.34$ and $59.11 \%$ with flow pumped of $1.26,0.99$ and $0.801 / \mathrm{min}$, obtaining a volume pumped per day of 1814.40, 1425.60 and 1152 liters.
\end{abstract}

Keywords: Hydraulic Ram, Ram pump, Renewable Energy, Clean Energy, Impulse valve. 


\section{Introducción}

En la actualidad en el ámbito rural la disponibilidad de agua o la energía para disponerla, es escasa, cara y muchas veces nula, en tal sentido se amerita la utilización de otras técnicas diferentes a la energía convencional, que ayude al poblador rural a disponer del agua a un bajo costo, siendo una alternativa viable la utilización de la bomba de ariete.

Una bomba de ariete hidráulico es un dispositivo, que usa la energía de una caída de agua, para generar potencia y poder elevar un porcentaje del agua de ingreso a una altura mayor que la altura de caída, de forma continua y automática sin ninguna otra energía externa,
El objetivo del presente trabajo es evaluar el rendimiento del ariete hidráulico modelo BAH 1.1/2, utilizando tubería de alimentación de PVC de 1.1/2" de diámetro y variando las longitudes en 1.0, 2.0 y $3.0 \mathrm{~m}$, para diferentes condiciones de operación de la válvula de impulso, variando la luz de la carreras de cierre en 3, 6 y $9 \mathrm{~mm}$.

\section{Revisión de literatura}

\section{Componentes del sistema hidráulico de la bomba de ariete}

Los componentes típicos para la operación del ariete hidráulico son los mostrados en la Figura 1.

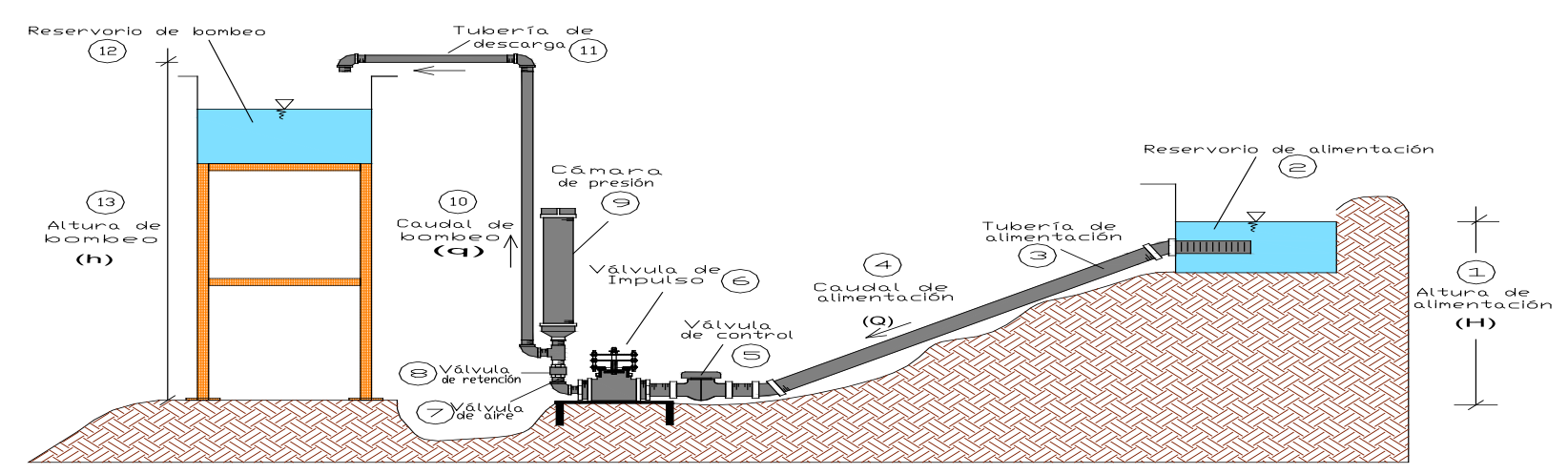

Figura 1. Componentes típicos del sistema hidráulico de la bomba de ariete.

Reservorio de alimentación (2): Constituye la fuente de suministro de agua y permite la entrada a la tubería de alimentación.

Tubería de alimentación (3): Conducto rígido relativamente corto, que cubre totalmente el salto hidráulico, conduciendo el agua desde el reservorio de alimentación hasta la válvula de impulso.

Válvula de control (5): Es una válvula que sirve para detener el flujo de ingreso, cuando se requiere parar la bomba de ariete en caso de efectuar mantenimiento.

Válvula de impulso (6): Elemento principal, válvula ubicada en el extremo aguas debajo de la tubería de alimentación, cuyo cierre instantáneo origina la sobrepresión que permite bombear parte del agua de alimentación del ariete, y cuya apertura permite la salida o alivio.

Válvula de aire (7): Un pequeño orificio ubicado en la zona de válvula, permite ingresar y restituir lentamente el aire que se pierde al mezclarse con el agua y salir junto con ella a través de la tubería de descarga.

Válvula de retención (8): Válvula de tipo check, localizada al ingreso de la cámara de aire, cuya apertura origina el paso de una parte del gasto de alimentación (Q) hacia la cámara de aire y cuyo cierre evita el flujo en sentido opuesto.

Cámara de presión (9): Ubicada inmediatamente por encima de la válvula de retención, el aire comprimido que en ella se confía, permite el ascenso del agua a través de la tubería de descarga, al actuar en forma similar a la de un resorte.

Tubería de descarga (11): Conducto que transporta el agua desde la cámara de aire hasta el reservorio de almacenamiento o sistema de distribución. No necesariamente esta línea de conducción tiene que ser rígida. 


\section{Funcionamiento de la bomba de ariete}

Se requiere de un desnivel de agua constante denominada altura de alimentación (1), como mínimo desde 1.0 metro de altura; que puede ser el agua proveniente de un rio, canal o manante, el agua debe ser sedimentada y filtrada para evitar que se obstruyan las válvulas. Al abrir la válvula de control (5) el agua del reservorio de almacenamiento (2) desciende por la tubería de alimentación (3) en una cantidad denominada caudal de alimentación (4) con elevada energía cinética, luego en un corto tiempo se cierra bruscamente la válvula de impulso (6). Esta detención repentina del flujo origina un aumento brusco o golpe de presión.

Por el principio de acción y reacción en la válvula de impulso (6) se originan sobre-presiones en sentido contrario. Por un lado, parte del agua pasa a través de la válvula de retención - check (8) a la cámara de presión, comprimiendo el aire de la cámara (9). Cuando se agota la energía de presión, se cierra la válvula de retención (8) y el aire de la cámara (9) se descomprime elevando el caudal (10) por la tubería de bombeo (11) hasta el reservorio (12) ubicado a la altura (13).

Al mismo tiempo que el agua ingresa a la cámara de presión (9) parte del agua retrocede por la tubería de alimentación (3), ocasionando una presión de succión en la válvula de impulso (5), que ayuda al mecanismo de la válvula (6) en abrir la misma, igualmente la presión de succión activa la válvula de aire (7) permitiendo el ingreso de aire al sistema que renueva el aire de la cámara (8), luego de este proceso se repite nuevamente el ciclo.

\section{Aspectos importantes para el diseño de la bomba de ariete}

Parámetros de la bomba de ariete: Los parámetros mecánicos de la operación no son muy entendibles. Algunos parámetros relacionados con la operación de la bomba de ariete se obtienen experimentalmente. Estos parámetros son:

- Longitud de la tubería de impulso (L);

- Área de la sección transversal de la tubería de impulso (A);

- Diámetro (D) y espesor de la tubería de impulso (e);

- Altura de caída (H);

- Altura de bombeo (h);

- Perdida por fricción en la tubería de impulso (hf);

- Perdida por fricción en la válvula de impulso (hm);

- Perdida por fricción en la válvula de retención (hm);

- La velocidad en la tubería de impulso, cuando la válvula de derrame comienza a cerrarse (Vo);

- La velocidad en flujo permanente (Vs) a través de la válvula completamente abierta;

- Peso de la válvula (W);

- Carrera de la válvula (S);

- El área del orificio de la válvula abierta (Ao);
- Área de la sección transversal de la válvula (Av) y;

- Tamaño de la cámara de aire.

Longitud de la tubería de Impulso: La tubería de impulso es un componente importante para la instalación de la bomba de ariete. La tubería de impulso debe ser capaz de soportar la alta presión originada por el cierre de la válvula de impulso. Relaciones empíricas para determinar la longitud de la tubería de impulso son:

$$
\begin{array}{ll}
6 H<L<12 H & \text { (Europa y Norte América) } \\
L=h+0.30(h / H) & (\text { Eytelwein) } \\
\mathrm{L}=900 \mathrm{H} /\left(\mathrm{N}^{\wedge} 2 * \mathrm{D}\right) & \text { (Russian) }
\end{array}
$$

Donde $\mathrm{N}$ es el número de golpes /minuto

$\mathrm{L}=150<\mathrm{L} / \mathrm{D}<1000 \quad$ (Calvert)

Muchos investigadores indican que la ecuación de Calvert da mejores resultados

Cámara de aire: Se recomienda que el volumen de la cámara de aire, sea 100 veces el volumen de agua bombeada por ciclo.

Válvula de aire: Experimentos con diferentes tamaños de válvula de aire, indican que no tiene mayor efecto en el funcionamiento de la bomba. Un agujero pequeño menor a $1 \mathrm{~mm}$ es suficiente.

Válvula de Impulso (derrame): El área de flujo de la válvula de derrame debe ser igual o mayor que el área de la sección de la tubería de impulso, para evitar retención del flujo.

Válvula de bombeo (Check): se recomienda $1.45 \mathrm{~cm}^{2} \mathrm{de}$ área por cada litro de agua bombeada.

Altura de bombeo (H): Con el simple peso de la válvula de impulso, la altura de caída no debe exceder $\operatorname{los} 4 \mathrm{~m}$, de otra manera la válvula se cerrara tan rápido y frecuentemente que no realizará ningún trabajo útil, en tal caso la válvula deberá llevar un resorte para regular el cierre.

\section{Potencia hidráulica}

Si se define Q como el caudal del fluido suministrado por un sistema, mecanismo o equipo hidráulico, se puede establecer que la potencia útil suministrada al fluido por estos medios es la energía por unidad de tiempo, quedando expresado la potencia hidráulica de la siguiente manera:

$$
P_{H}=\gamma Q H
$$

Donde:

$\mathrm{P}_{\mathrm{H}} \quad$ Potencia hidráulica, Potencia suministrada al fluido (Vatios)

$\gamma \quad$ Peso específico del fluido $\left(\mathrm{N} / \mathrm{m}^{3}\right)$

Q Caudal suministrado al fluido $\left(\mathrm{m}^{3} / \mathrm{s}\right)$

$\mathrm{H} \quad$ Energía de elevación o presión suministrada al 
fluido $(\mathrm{m})$

\section{Relaciones empíricas de eficiencia de ariete hidráulico}

Hay dos métodos comúnmente utilizados para calcular la eficiencia del ariete hidráulico, la de Rankine y la D’Aubuisson, métodos mostrados en las ecuaciones:

$\mathrm{E}($ Rankine $)=\left(Q^{*} h\right) /((Q+Q w) * H) \times 100$

$\mathrm{E}\left(D^{\prime}\right.$ Aubuisson $)=\left(Q^{*} H d\right) /((Q+Q w) * \mathrm{H}) \times 100$

Donde:

E es la eficiencia de la bomba de ariete (\%)

Q es el flujo bombeado (1/min)

Qw es el flujo desperdiciado ( $1 / \mathrm{min})$

$\mathrm{h} \quad$ es la altura de bombeo encima del ariete (m)

$\mathrm{H} \quad$ es la altura de caída por encima de la válvula de derrame (m)

Hd es la altura total por encima de la válvula de derrame $=(\mathrm{H}+\mathrm{h})(\mathrm{m})$

\section{Relación de eficiencia de fabricación de ariete hidráulico}

La mayoría de fabricantes toman una eficiencia simplificada, como la relación de potencias hidráulicas entregada por el ariete hidráulico respecto al suministrado al mismo, esta relación está dada como:

$\mathrm{E}($ Fabricación $)=\left({ }^{\gamma} \mathrm{q} \mathrm{h}\right) /\left({ }^{\gamma} \mathrm{QH}\right) \times 100$

\section{Dónde:}

E es la eficiencia de la bomba de ariete (\%)

q Caudal bombeado ( $1 / \mathrm{min})$

h Altura bombeada (m)

Q Caudal de alimentación ( $1 / \mathrm{min})$

$\mathrm{H} \quad$ altura de alimentación (m)

\section{Materiales y métodos}

La evaluación del ariete hidráulico fue realizada en el Laboratorio de Hidráulica y Mecánica de Fluidos del Departamento de Recursos Hídricos de la Facultad de Ingeniería Agrícola de la Universidad Nacional Agraria La Molina.

\section{Banco de prueba}

El banco de prueba es una estructura de material noble de $5.18 \times 1.14 \times 0.80 \mathrm{~m}$ de largo, ancho y alto respectivamente, consta de dos cuerpos en sentido longitudinal y con sistema re-circulante de flujo de agua, Como reservorio de alimentación se utilizó un tanque fabricado en tubería PVC 110 mm (4") de diámetro y $3.0 \mathrm{~m}$ de altura, anclado y fijado en forma vertical, el cual se abastecía de agua con una electrobomba Pedrollo modelo CPm 610X de 0.85 $\mathrm{HP}(0.60 \mathrm{~kW})$ de $10-80 \mathrm{l} / \mathrm{min}$ de caudal, y de $12-30$ $\mathrm{m}$ de presión. Con el sistema de abastecimiento, parte del agua bombeada fue derivada a la cámara de alimentación y está a la tubería de alimentación y el agua en exceso retornaba por una tubería de derivación al tanque abastecedor.
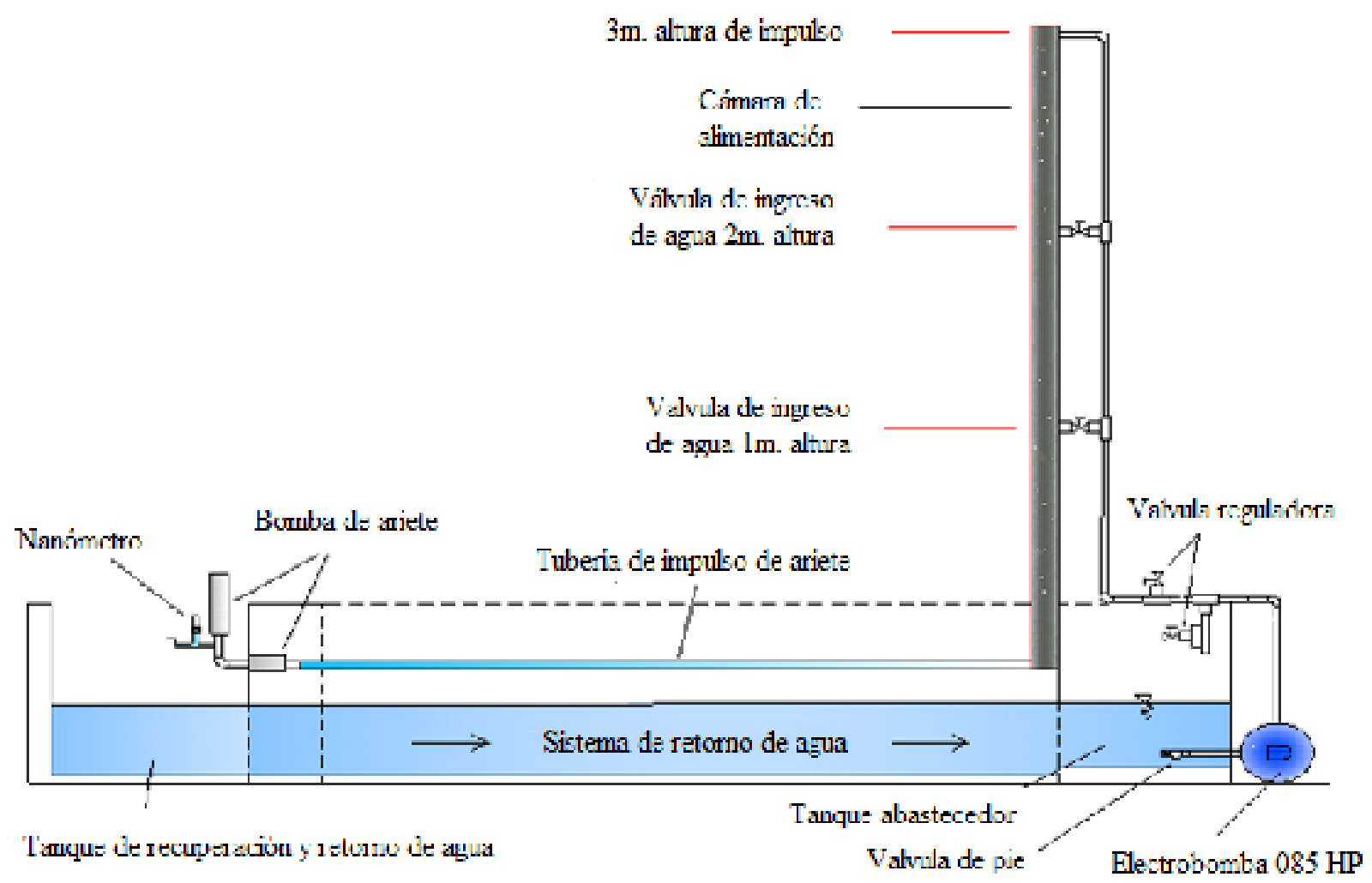

Figura 2. Esquema del banco de prueba utilizado. 


\section{Bomba de ariete hidráulico BAH 1.1/.2}

La bomba de ariete utilizada para la prueba fue del modelo BAH-1.1/2 alimentada con tubería de PVC $40 \mathrm{~mm}(1.1 / 2$ ") y con salida de tubería de FG $21 \mathrm{~mm}$ $(1 / 2$ ") de diámetro respectivamente, bomba ensamblada principalmente con accesorios comerciales de fierro galvanizado, acero inoxidable y bronce, como se muestra en las Figuras. 3 y 4 y el detalle de los componentes en la Tabla 1.

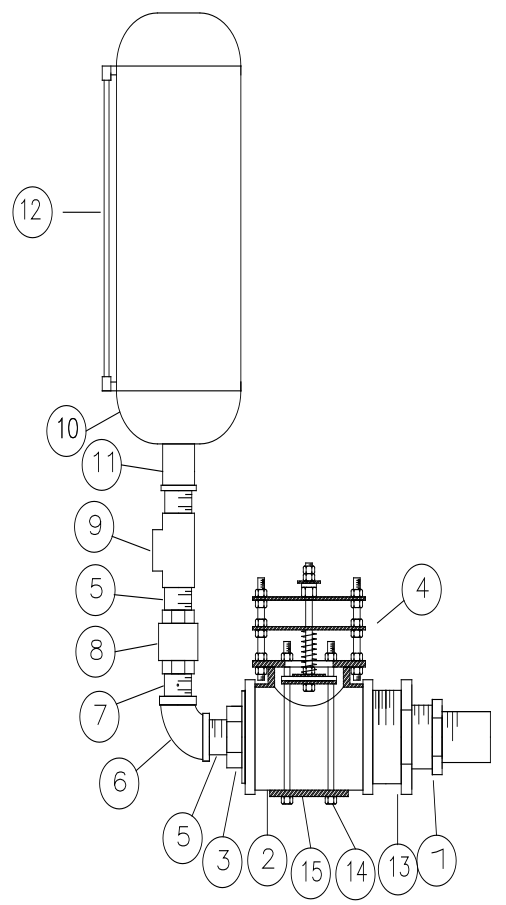

Figura 3. Esquema de los componentes de la bomba de ariete hidráulico BAH 1.1/2.

Tabla 1. Elementos y componentes de la bomba de ariete hidráulico BAH 1.1/2.

\begin{tabular}{|c|c|c|c|}
\hline Ítem & Descripción & Cantidad & Material \\
\hline 1 & Bushing de 2 a 1 1/2" & 1 & $\mathrm{Fe}$ \\
\hline 2 & Tee de $3 \times 3$ “ & 1 & $\mathrm{Fe}$ \\
\hline 3 & Bushing de 2 a $1 "$ & 1 & $\mathrm{Fe}$ \\
\hline 4 & Válvula de impulso de $11 / 2 "$ & 1 & $\mathrm{Fe}$ \\
\hline 5 & Niple de 1 x $1 "$ & 3 & $\mathrm{Fe}$ \\
\hline 6 & Codo $90^{\circ} \times 1 "$ & 1 & $\mathrm{Fe}$ \\
\hline 7 & $\begin{array}{l}\text { Válvula de aire de } 1 " \text { con } \\
\text { agujero de } 1 / 16 "\end{array}$ & 1 & $\mathrm{Fe}$ \\
\hline 8 & $\begin{array}{l}\text { Válvula Check vetical con } \\
\text { resorte de } 1 \text { "dia }\end{array}$ & 1 & Bronce \\
\hline 9 & Tee de $1 \times 1 "$ & 1 & $\mathrm{Fe}$ \\
\hline 10 & Cámara de 3 x 16" & 1 & $\mathrm{Fe}$ \\
\hline 11 & $\begin{array}{l}\text { Unión cilíndrica de } 1 \text { " rosca, } \\
60.5 \mathrm{~mm} \text { esp }\end{array}$ & 1 & $\mathrm{Fe}$ \\
\hline 12 & Manguera de 1/4" dia & 1 & $\mathrm{Ny}$ \\
\hline 13 & Bushing de 3 a $2 "$ & 1 & $\mathrm{Fe}$ \\
\hline 14 & Perno de 5/16 x $7 "$ & 1 & $\mathrm{Fe}$ \\
\hline 15 & Plancha circular: $148 \times 5 \mathrm{~mm}$ & 1 & $\mathrm{Fe}$ \\
\hline
\end{tabular}

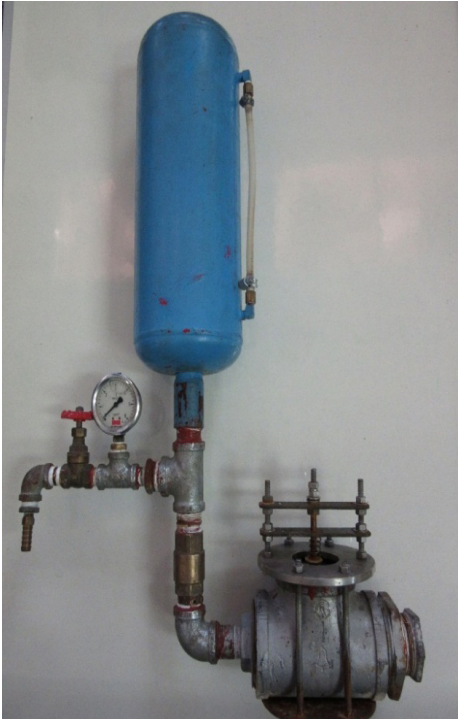

Figura 4. Fotografía frontal de la bomba de ariete hidráulico BAH 1.1/2.

\section{Válvula de impulso}

Elemento principal del ariete hidráulico, válvula tipo puente construido con platinas y espárragos que permiten regular la presión por medio de un resorte, el eje de la válvula es un esparrago que soporta un disco como elemento de cierre en acero inoxidable con base de caucho vulcanizado, todos estos componentes son montados excéntricamente sobre un disco de mayor diámetro en acero inoxidable, como se muestra en las Figuras. 5 y 6 y la relación de sus componentes en la Tabla 2.

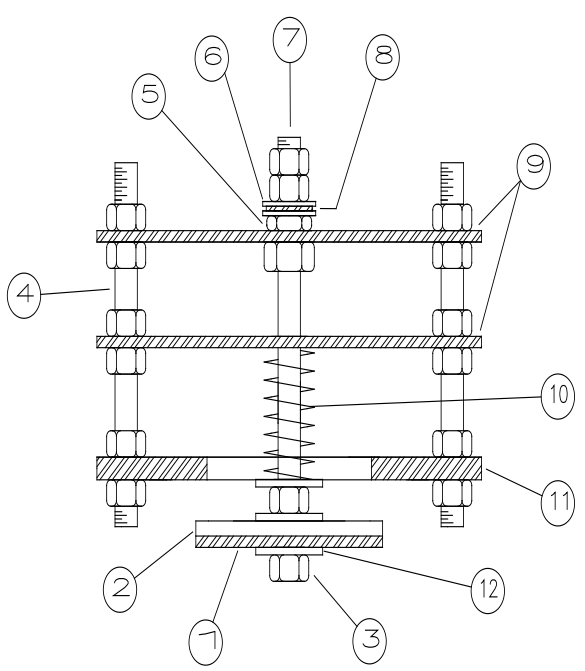

Figura 5. Esquema de la válvula de impulso de 1.1/2”.

Tabla 2. Relación de componentes de la válvula de impulso de 1.1/2.

\begin{tabular}{clcc}
\hline Ítem & \multicolumn{1}{c}{ Descripción } & Cantidad & Material \\
\hline 1 & $\begin{array}{l}\text { Disco } 73 \mathrm{~mm} \text { Dext } \\
\text { Dint, 3mm esp }\end{array}$ & 1 & Fe inox \\
\hline
\end{tabular}




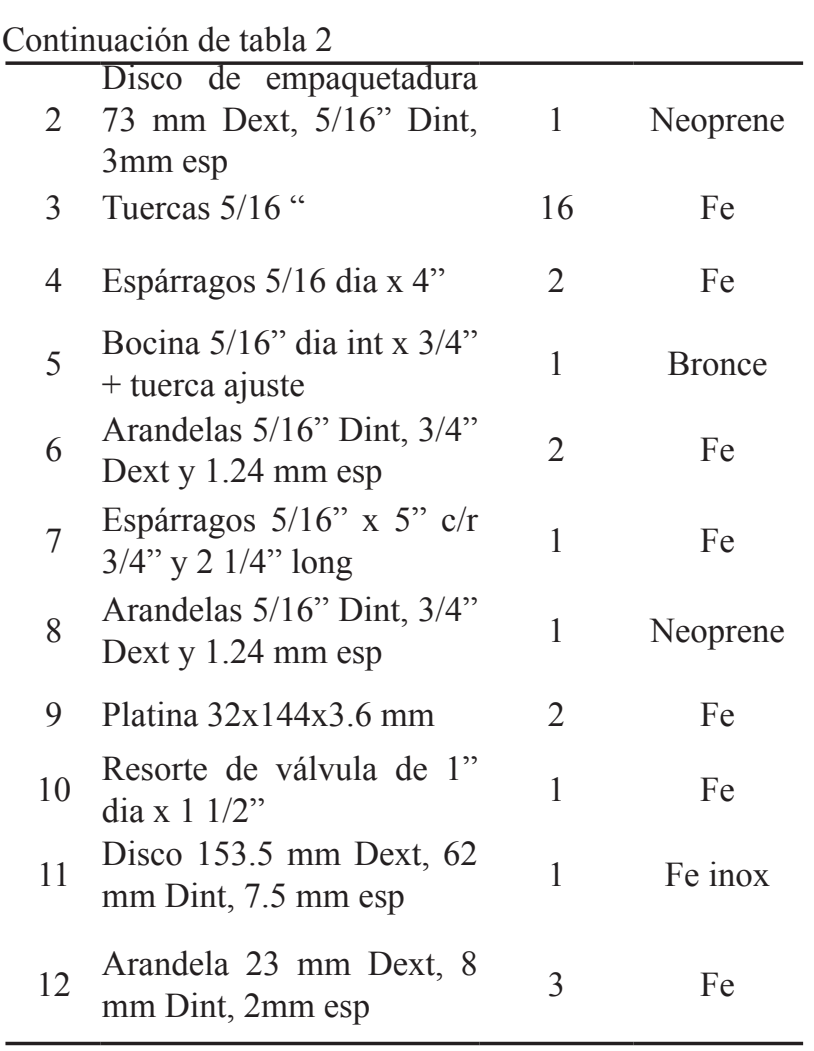

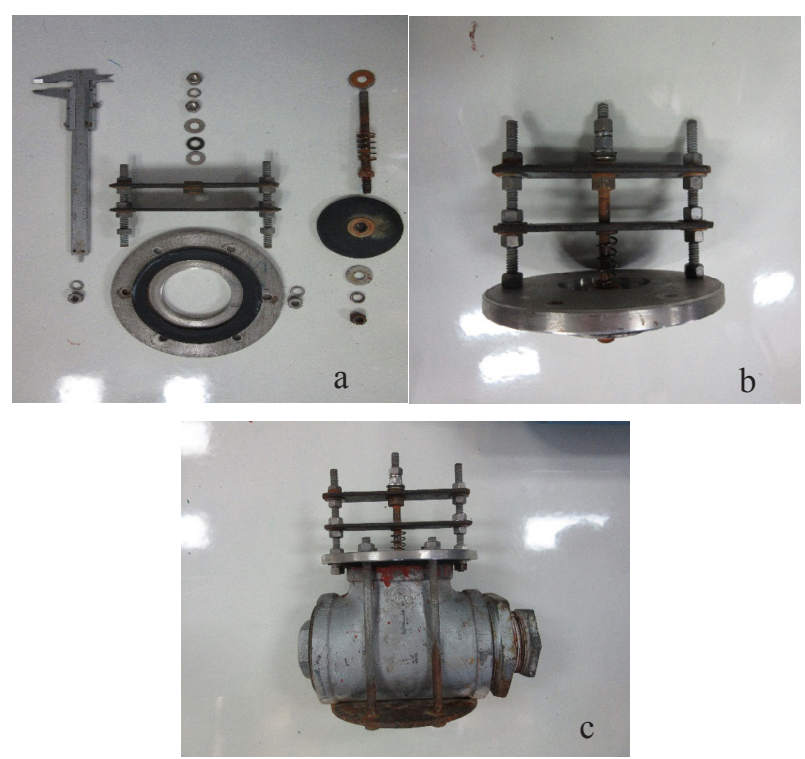

Figura 6. Despiece (a), armado (b) y montaje (c) de la válvula de impulso.

\section{Herramientas}

Las principales herramientas utilizadas para el montaje y desmontaje de la bomba de ariete así como para la calibración y regulación de la válvula de impulso durante la prueba de evaluación son los mostrados en la Figura 7.

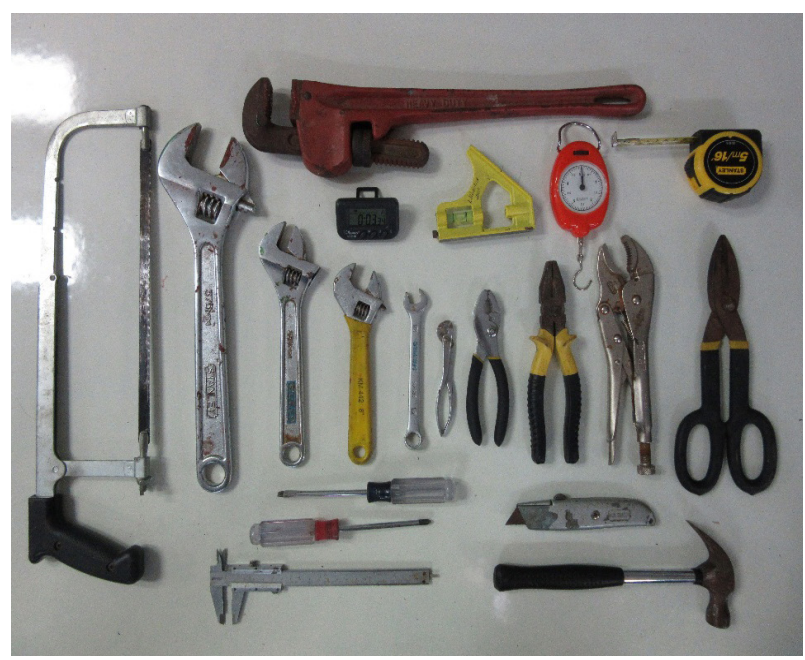

Figura 7. Herramientas utilizadas para el montaje y desmontaje de la bomba de ariete.

\section{Procedimiento}

\section{Ensayos preliminares}

Se efectuaron ensayos preliminares a fin de fijar los parámetros iniciales de operación y prueba de la bomba de ariete BAH 1.1/2, estos ensayos permitieron definir los siguientes parámetros: a). diámetro de paso de válvula $62 \mathrm{~mm}$, mayor al diámetro interior de la tubería de alimentación de $40 \mathrm{~mm}$, con el propósito de reducir el tiempo de cierre de la válvula de impulso, b). el peso en el vástago más accesorios y tensión del resorte de la válvula de cierre se fijó en 1500 gramos a fin de prolongar el tiempo de abierta de la válvula, c). la carrera inicial de luz de cierre de válvula $6 \mathrm{~mm}$ próximo al valor de ideal de $6.5 \mathrm{~mm}$ que hace equivalente el área de paso de la válvula al área de la sección transversal de la tubería de alimentación, d). se probaron con diferentes alturas de alimentación, definiéndose finalmente en $1.0 \mathrm{~m}$.

\section{Ensayos definitivos}

De acuerdo a los objetivos y parámetros de operación se definieron las siguientes condiciones de evaluación final, se optó para la prueba un desnivel de alimentación $1.0 \mathrm{~m}$ entre el nivel del agua en el reservorio de alimentación y el ariete hidráulico mismo, este desnivel fue mantenido constante durante toda la prueba con la electrobomba.

Para obtener las situaciones de 1.0, 2.0 y $3.0 \mathrm{~m}$ de longitud de tubería, desde el ariete respecto a la cámara de alimentación, se utilizó tres tuberías de PVC con las respectivas longitudes y acoples, se usaron ángulos ranurados de fierro tanto para fijar el ariete mismo, como para fijar las diferentes tuberías con abrazaderas de 1.1/2" cada $0.5 \mathrm{~m}$.

Para cada longitud de tubería de prueba de 1.0, 2.0 y 3.0 $\mathrm{m}$, se modificó la luz de cierre de la válvula de impuso (carrera $\mathrm{S}$ ), con valores de 3, 6 y $9 \mathrm{~mm}$ respectivamente, bajo estas condiciones se realizó la prueba registrándose valores de presión de bombeo (h), caudal de bombeo (q) 
y caudal de alimentación (Q).Un manómetro de glicerina de 0 a $6 \mathrm{~kg} / \mathrm{cm} 2$, fue utilizado para medir la presión de bombeo; y las respectivas cantidades de los caudales de bombeo (q) y alimentación (Q) se determinaron efectuando un aforo volumétrico para lo cual se utilizó recipientes y cronometro.

\section{Resultados y discusión}

Los resultados de las pruebas de evaluación realizadas al ariete hidráulico BAH 1.1/2, con longitudes de la tubería de alimentación de 1, 2 y 3 m en PVC, para diferentes condiciones de carrera de la válvula de impulso en 3,6 y 9 mm, se presentan en las Tablas 3, 4 y 5. Así mismo las respectivas gráficas del caudal bombeado vs. Altura de bombeo, Eficiencia y Caudal de alimentación, para las diferentes longitudes de tuberías con las respectivas carreras se presentan en las gráficas de las Figuras. 8 al 16.

Tabla 3. Resultados de la Prueba del Ariete Hidráulico BAH 1.1/2 con Tubería de PVC de 1, 2 y 3 m de longitud y 3 mm de carrera de válvula de impulso.

\begin{tabular}{|c|c|c|c|c|c|c|c|c|}
\hline $\begin{array}{c}\text { Longitud de } \\
\text { Tuberia L(m) }\end{array}$ & $\begin{array}{c}\text { Altura de } \\
\text { Bombeo } h(m)\end{array}$ & $\begin{array}{c}\text { Caudal de } \\
\text { Alimentación Q } \\
\text { (lt/min) }\end{array}$ & $\begin{array}{l}\text { Caudal de } \\
\text { Bombeo } \\
\text { q(lt/min) }\end{array}$ & $\begin{array}{c}\text { Caudal de derrame } \\
\text { Q-q (lt } / \mathrm{min}) .\end{array}$ & $\begin{array}{c}\text { Eficiencia } \\
\text { n(\%) }\end{array}$ & $q / Q \times 100$ & $\begin{array}{c}\text { Golpes por } \\
\text { Minuto GPM }\end{array}$ & $\begin{array}{c}\text { Potencia } \\
\text { Watts }\end{array}$ \\
\hline \multirow{5}{*}{1} & 30 & 19.09 & 0.00 & 19.09 & 0.00 & 0.00 & 255 & 0.00 \\
\hline & 25 & 31.06 & 0.24 & 30.82 & 19.00 & 0.76 & 255 & 0.96 \\
\hline & 20 & 33.19 & 0.40 & 32.79 & 24.10 & 1.21 & 244 & 1.31 \\
\hline & 15 & 33.72 & 1.06 & 32.65 & 47.34 & 3.16 & 244 & 2.61 \\
\hline & 10 & 34.18 & 1.59 & 32.59 & 46.52 & 4.65 & 244 & 2.60 \\
\hline \multirow{7}{*}{2} & 35 & 20.43 & 0.00 & 20.43 & 0.00 & 0.00 & 116 & 0.00 \\
\hline & 30 & 32.31 & 0.42 & 31.88 & 39.37 & 1.31 & 140 & 2.08 \\
\hline & 25 & 31.13 & 0.47 & 30.65 & 38.02 & 1.52 & 132 & 1.93 \\
\hline & 20 & 30.55 & 0.58 & 29.96 & 38.06 & 1.90 & 133 & 1.90 \\
\hline & 15 & 32.74 & 1.05 & 31.70 & 47.95 & 3.20 & 134 & 2.57 \\
\hline & 10 & 37.35 & 1.93 & 35.41 & 51.77 & 5.18 & 148 & 3.16 \\
\hline & 8 & 33.86 & 2.19 & 31.67 & 51.66 & 6.46 & 156 & 2.86 \\
\hline \multirow{7}{*}{3} & 38 & 17.91 & 0.00 & 17.91 & 0.00 & 0.00 & 87 & 0.00 \\
\hline & 30 & 29.69 & 0.42 & 29.27 & 42.31 & 1.41 & 108 & 2.05 \\
\hline & 25 & 32.18 & 0.63 & 31.55 & 49.20 & 1.97 & 111 & 2.59 \\
\hline & 20 & 32.19 & 0.79 & 31.41 & 48.87 & 2.44 & 112 & 2.57 \\
\hline & 15 & 32.00 & 1.21 & 30.79 & 56.88 & 3.79 & 111 & 2.98 \\
\hline & 10 & 35.08 & 2.13 & 32.95 & 60.63 & 6.06 & 117 & 3.48 \\
\hline & 6 & 35.31 & 3.59 & 31.72 & 61.00 & 10.17 & 105 & 3.52 \\
\hline
\end{tabular}

Tabla 4. Resultados de la Prueba del Ariete Hidráulico BAH 1.1/2 con Tubería de PVC de 1, 2 y 3 m de longitud y 6 mm de carrera de válvula de impulso.

\begin{tabular}{|c|c|c|c|c|c|c|c|c|}
\hline $\begin{array}{c}\text { Longitud de } \\
\text { Tuberia L(m) }\end{array}$ & $\begin{array}{c}\text { Altura de } \\
\text { Bombeo h(m) }\end{array}$ & $\begin{array}{c}\text { Caudal de } \\
\text { Alimentación Q } \\
\text { (It/min) }\end{array}$ & $\begin{array}{c}\text { Caudal de } \\
\text { Bombeo } \\
\text { q(lt/min) } \\
\end{array}$ & $\begin{array}{c}\text { Caudal de derrame } \\
\text { Q-q (lt/min). }\end{array}$ & $\begin{array}{c}\text { Eficiencia } \\
\mathbf{n}(\%) \\
\end{array}$ & $q / Q \times 100$ & $\begin{array}{c}\text { Golpes por } \\
\text { Minuto GPM } \\
\end{array}$ & $\begin{array}{c}\text { Potencia } \\
\text { Watts }\end{array}$ \\
\hline \multirow[t]{5}{*}{ क } & 30 & 26.52 & 0.00 & 26.52 & 0.00 & 0.00 & 157 & 0.00 \\
\hline & 25 & 37.56 & 0.30 & 37.26 & 19.97 & 0.80 & 193 & 1.23 \\
\hline & 20 & 37.15 & 0.49 & 36.66 & 26.13 & 1.31 & 226 & 1.59 \\
\hline & 15 & 37.91 & 1.01 & 36.91 & 39.83 & 2.66 & 227 & 2.47 \\
\hline & 10 & 43.67 & 1.90 & 41.77 & 43.51 & 4.35 & 223 & 3.11 \\
\hline \multirow{8}{*}{2} & 46 & 29.33 & 0.00 & 29.33 & 0.00 & 0.00 & 94 & 0.00 \\
\hline & 40 & 34.83 & 0.14 & 34.70 & 15.77 & 0.39 & 109 & 0.90 \\
\hline & 35 & 37.64 & 0.30 & 37.34 & 28.14 & 0.80 & 121 & 1.73 \\
\hline & 30 & 37.72 & 0.43 & 37.29 & 34.36 & 1.15 & 119 & 2.12 \\
\hline & 25 & 36.60 & 0.66 & 35.94 & 44.90 & 1.80 & 105 & 2.69 \\
\hline & 20 & 34.47 & 0.70 & 33.77 & 40.54 & 2.03 & 108 & 2.28 \\
\hline & 15 & 38.09 & 1.15 & 36.94 & 45.42 & 3.03 & 115 & 2.83 \\
\hline & 10 & 42.34 & 2.36 & 39.98 & 55.74 & 5.57 & 120 & 3.86 \\
\hline \multirow{10}{*}{3} & 50 & 21.58 & 0.00 & 21.58 & 0.00 & 0.00 & 77 & 0.00 \\
\hline & 45 & 26.58 & 0.07 & 26.51 & 12.53 & 0.28 & 81 & 0.54 \\
\hline & 40 & 32.02 & 0.23 & 31.79 & 29.31 & 0.73 & 90 & 1.53 \\
\hline & 35 & 35.26 & 0.41 & 34.85 & 41.03 & 1.17 & 96 & 2.37 \\
\hline & 30 & 38.80 & 0.63 & 38.17 & 48.96 & 1.63 & 96 & 3.11 \\
\hline & 25 & 36.51 & 0.67 & 35.85 & 45.65 & 1.83 & 98 & 2.73 \\
\hline & 20 & 36.80 & 1.01 & 35.80 & 54.63 & 2.73 & 95 & 3.29 \\
\hline & 15 & 40.82 & 1.57 & 39.25 & 57.58 & 3.84 & 101 & 3.84 \\
\hline & 10 & 42.13 & 2.36 & 39.77 & 55.93 & 5.59 & 105 & 3.85 \\
\hline & 6 & 38.93 & 4.43 & 34.50 & 68.29 & 11.38 & 104 & 4.35 \\
\hline
\end{tabular}


Tabla 5. Resultados de la Prueba del Ariete Hidráulico BAH 1.1/2 con Tubería de PVC de 1, 2 y 3 m de longitud y 9 mm de carrera de válvula de impulso.

\begin{tabular}{|c|c|c|c|c|c|c|c|c|}
\hline $\begin{array}{c}\text { Longitud de } \\
\text { Tuberia L(m) } \\
\end{array}$ & $\begin{array}{c}\text { Altura de } \\
\text { Bombeo } h(m) \\
\end{array}$ & $\begin{array}{c}\text { Caudal de } \\
\text { Alimentación Q } \\
\text { (lt } / \mathbf{m i n})\end{array}$ & $\begin{array}{c}\text { Caudal de } \\
\text { Bombeo } \\
\text { q(It/min) } \\
\end{array}$ & $\begin{array}{c}\text { Caudal de derrame } \\
\text { Q-q (lt/min). }\end{array}$ & $\begin{array}{c}\text { Eficiencia } \\
\mathbf{n}(\%) \\
\end{array}$ & $q / Q \times 100$ & $\begin{array}{c}\text { Golpes por } \\
\text { Minuto GPM } \\
\end{array}$ & $\begin{array}{c}\text { Potencia } \\
\text { Watts }\end{array}$ \\
\hline \multirow{5}{*}{1} & 30 & 27.01 & 0.00 & 27.01 & 0.00 & 0.00 & 152 & 0.00 \\
\hline & 25 & 36.56 & 0.41 & 36.15 & 27.90 & 1.12 & 189 & 1.67 \\
\hline & 20 & 38.51 & 0.52 & 37.99 & 27.21 & 1.36 & 209 & 1.71 \\
\hline & 15 & 43.17 & 1.11 & 42.06 & 38.68 & 2.58 & 220 & 2.73 \\
\hline & 10 & 43.79 & 1.62 & 42.17 & 37.00 & 3.70 & 216 & 2.65 \\
\hline \multirow{10}{*}{2} & 46 & 27.09 & 0.00 & 27.09 & 0.00 & 0.00 & 100 & 0.00 \\
\hline & 40 & 37.58 & 0.21 & 37.38 & 21.85 & 0.55 & 116 & 1.34 \\
\hline & 40 & 35.27 & 0.20 & 35.07 & 22.68 & 0.57 & 116 & 1.31 \\
\hline & 35 & 41.18 & 0.25 & 40.93 & 21.08 & 0.60 & 120 & 1.42 \\
\hline & 30 & 41.67 & 0.52 & 41.15 & 37.63 & 1.25 & 120 & 2.56 \\
\hline & 25 & 36.34 & 0.61 & 35.73 & 42.01 & 1.68 & 120 & 2.50 \\
\hline & 20 & 37.28 & 0.63 & 36.65 & 33.62 & 1.68 & 112 & 2.05 \\
\hline & 15 & 41.35 & 1.31 & 40.04 & 47.40 & 3.16 & 116 & 3.20 \\
\hline & 10 & 47.53 & 2.27 & 45.26 & 47.83 & 4.78 & 120 & 3.72 \\
\hline & 7 & 45.92 & 3.04 & 42.88 & 46.34 & 6.62 & 120 & 3.48 \\
\hline \multirow{10}{*}{3} & 52 & 23.53 & 0.00 & 23.53 & 0.00 & 0.00 & 72 & 0.00 \\
\hline & 45 & 31.24 & 0.19 & 31.05 & 27.66 & 0.61 & 76 & 1.41 \\
\hline & 40 & 33.56 & 0.31 & 33.25 & 36.87 & 0.92 & 80 & 2.02 \\
\hline & 35 & 37.41 & 0.48 & 36.94 & 44.53 & 1.27 & 80 & 2.72 \\
\hline & 30 & 40.60 & 0.80 & 39.80 & 59.11 & 1.97 & 81 & 3.92 \\
\hline & 25 & 40.88 & 0.99 & 39.90 & 60.34 & 2.41 & 84 & 4.03 \\
\hline & 20 & 39.29 & 1.26 & 38.03 & 64.16 & 3.21 & 86 & 4.12 \\
\hline & 15 & 43.65 & 1.96 & 41.69 & 67.35 & 4.49 & 90 & 4.81 \\
\hline & 10 & 44.50 & 2.67 & 41.83 & 59.92 & 5.99 & 92 & 4.36 \\
\hline & 6 & 42.95 & 3.80 & 39.15 & 53.09 & 8.85 & 92 & 3.73 \\
\hline
\end{tabular}

\section{Caudal de Bombeo vs. Presión y/o Altura de Bombeo}

En la gráfica de la Figura 8, y las Tabla 3, para carrera de válvula $\mathrm{S}=3 \mathrm{~mm}$, se puede observar que la bomba de ariete evaluada con longitudes de tuberías de $\mathrm{L}=1, \mathrm{~L}=2$ y $\mathrm{L}=3 \mathrm{~m}$, alcanzaron respectivamente presiones máximas de 30,35 y 38 mca con descarga nula, y se obtuvieron descargas máximas de $1.59,1.93$ y $2.13 \mathrm{l} / \mathrm{min}$ a presiones de trabajo de 10 mca.

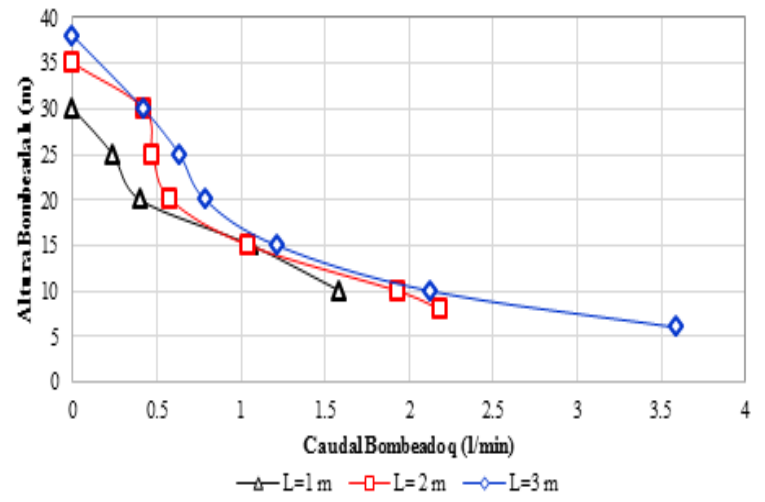

Figura 8.- Caudal de Bombeo rs. Altura de Bombeo de BAH 1.1/2 Para longitudes de tuberias de prueba, con Carrera $S=3 \mathrm{~mm}$

En la Figura 9, y Tabla 4, para carrera de válvula a $S=6$ $\mathrm{mm}$ y en las mismas condiciones de longitud de tubería de $\mathrm{L}=1, \mathrm{~L}=2$ y $\mathrm{L}=3 \mathrm{~m}$, alcanzaron presiones máximas de 30, 46 y 50 mca con descarga nula, y se obtuvieron descargas máximas de $1.90,2.36$ y $2.36 \mathrm{l} / \mathrm{min}$ a presiones de trabajo de 10 mca.

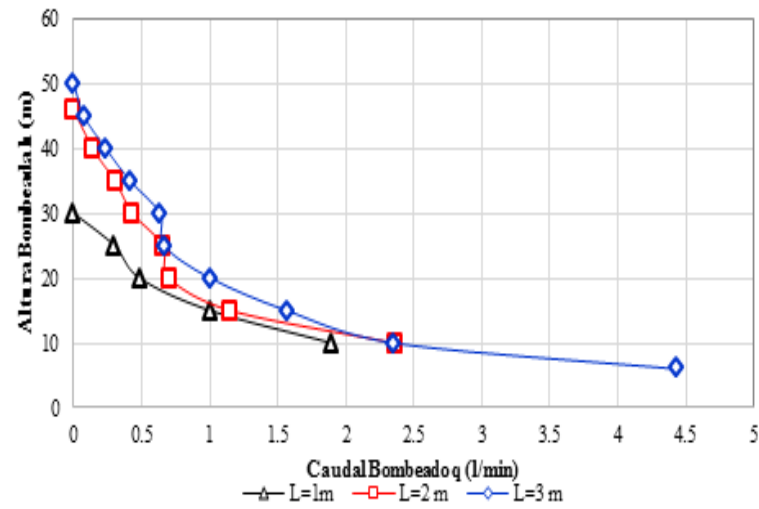

Figura 9.- Caudal de Bombeo rs. Altura de Bombeo de BAH 1.1/2 Para longitudes de tuberias de prueba, con Carrera $S=6 \mathrm{~mm}$

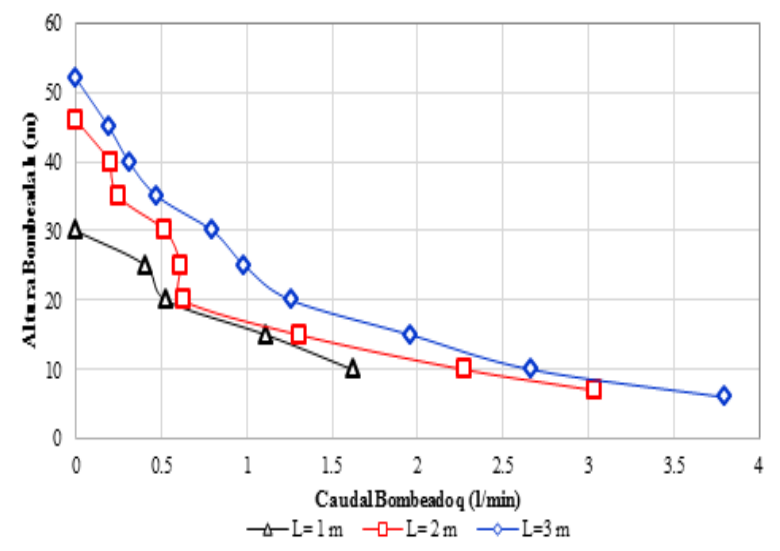

Figura 10.- Caudal de Bombeo vs. Altura de Bombeo de BAH 1.1/2 Para longitudes de tuberias de prueba, con Carrera $\mathrm{S}=9 \mathrm{~mm}$ 
En la Figura 10, y Tabla 5, para carrera de válvula a $S=9$ $\mathrm{mm}$ y bajo las mismas condiciones de longitud de tubería y, alcanzaron presiones máximas de 30, 46 y 52 mca con descarga nula, y se obtuvieron descargas máximas de $1.62,2.27$ y $2.67 \mathrm{l} / \mathrm{min}$ a presiones de trabajo de $10 \mathrm{mca}$. Finalmente, según las gráficas de las Figuras. 8, 9 y 10, y las Tablas 3, 4 y 5 las alturas máximas alcanzadas en diferentes carrera de válvula de impulso con $S=3, S=6$ y $\mathrm{S}=9 \mathrm{~mm}$, resulto en la tubería de mayor longitud $\mathrm{L}=3$ $\mathrm{m}$, Siendo la altura de bombeo máxima de 38,50 y $52 \mathrm{~m}$ a en las distintas carreras con caudal de bombeo nulo y descargas elevadas de $2.13,2.36$ y $2.67 \mathrm{l} / \mathrm{min}$ a $10 \mathrm{~m}$ de presión de trabajo.

\section{Caudal de Bombeo vs. Eficiencia}

En la Figura 11, y las Tabla 3, para carrera de válvula $\mathrm{S}=3 \mathrm{~mm}$, se puede observar que la bomba de ariete evaluada con longitudes de tuberías de $\mathrm{L}=1, \mathrm{~L}=2$ y $\mathrm{L}=3$ $\mathrm{m}$, alcanzaron respectivamente eficiencias elevadas de $46.52,51.77$ y $60.63 \%$, obtenidas en alturas de bombeo como 10,10 y 10 mca a caudales bombeo de $1.59,1.93$ y $2.13 \mathrm{l} / \mathrm{min}$. Respectivamente según longitud de tubería.

En la Figura 12, y las Tabla 4, para carrera de válvula $\mathrm{S}=6 \mathrm{~mm}$, se puede observar que la bomba de ariete evaluada con longitudes de tuberías de $\mathrm{L}=1, \mathrm{~L}=2$ y $\mathrm{L}=3$ $\mathrm{m}$, alcanzaron respectivamente eficiencias elevadas de $43.51,55.74$ y $55.93 \%$, obtenidas en alturas de bombeo como 10,10 y 10 mca a caudales bombeo de 1.90, 2.36 y $2.36 \mathrm{l} / \mathrm{min}$. Respectivamente según longitud de tubería.

En la Figura 13, y las Tabla 5, para carrera de válvula $\mathrm{S}=9 \mathrm{~mm}$, se puede observar que la bomba de ariete evaluada con longitudes de tuberías de $\mathrm{L}=1, \mathrm{~L}=2$ y $\mathrm{L}=3$ $\mathrm{m}$, alcanzaron respectivamente eficiencias elevadas de $37.0,47.83$ y $59.92 \%$, obtenidas en alturas de bombeo como 10,10 y 10 mca a caudales bombeo de 1.62, 2.27 y $2.67 \mathrm{l} / \mathrm{min}$. Respectivamente según longitud de tubería.

Finalmente, según las gráficas de las Figuras. 11, 12 y 13, y las Tablas 3, 4 y 5 las eficiencias mayores alcanzadas en diferentes carrera de válvula de impulso con $S=3, S=6$ y $\mathrm{S}=9 \mathrm{~mm}$, fueron dadas por la tubería de mayor longitud $\mathrm{L}=3 \mathrm{~m}$, siendo estas $60.63,55.93$ y $59.92 \%$ con caudales de bombeo de $2.13,2.36$ y $2.671 / \mathrm{min}$

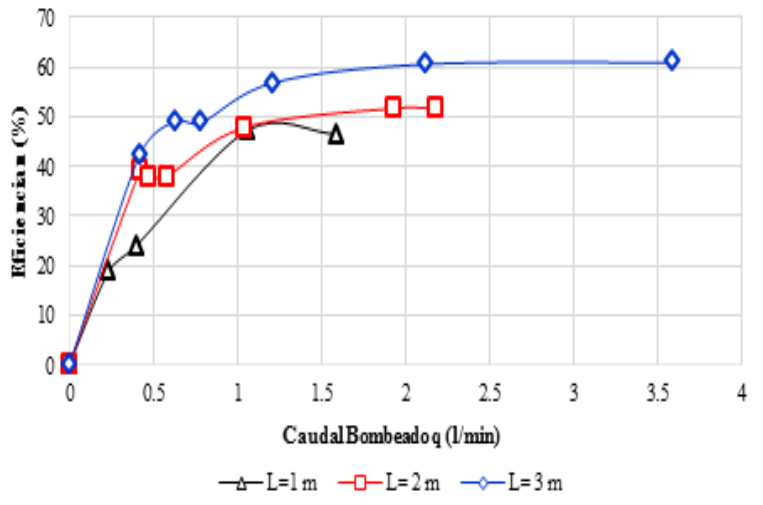

Figura 11.- Caudal de Bombeo is. Fficiencia de BAH 1.1/2 Para longitudes de tuberias de prueba, con Carrera $\mathrm{S}=3 \mathrm{~mm}$

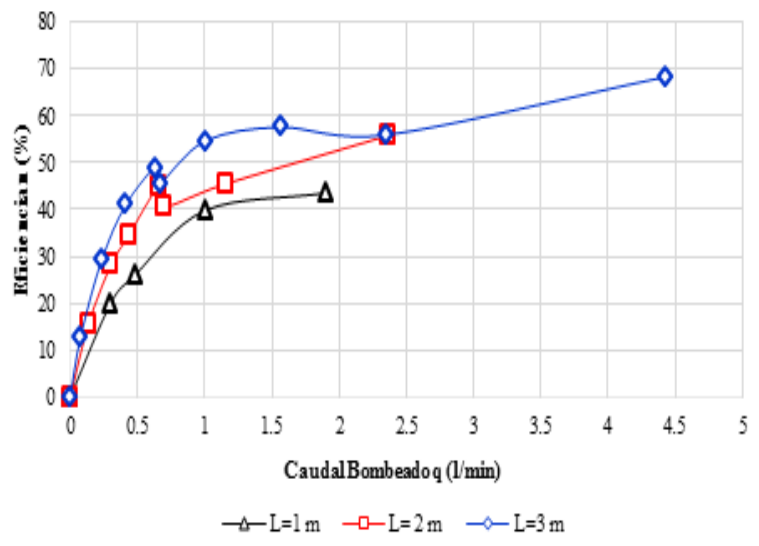

Figura 12.-Caudal de Bombeo is. Fficiencia de BAH 1.1/2 Para longitudes de tuberias de prueba, con Carrera $\mathrm{S}=6 \mathrm{~mm}$

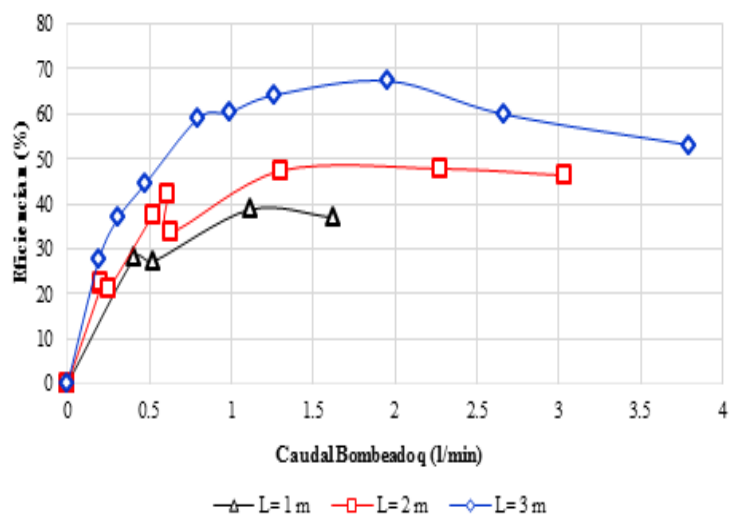

Figura 13.- Caudal de Bombeo rs. Eficiencia de BAH 1.1/2 Para longitudes de tuberias de prueba, Carrera $S=9 \mathrm{~mm}$

\section{Caudal de Bombeo vs. Caudal de alimentación}

En la Figura 14, y las Tabla 3, para carrera de válvula $S=3$ $\mathrm{mm}$, se puede observar que la bomba de ariete evaluada con longitudes de tuberías de $\mathrm{L}=1, \mathrm{~L}=2$ y $\mathrm{L}=3 \mathrm{~m}$, requiere respectivamente mayor caudal de alimentación en 34.18, 37.35 y $35.08 \mathrm{l} / \mathrm{min}$ obtenidas en alturas de bombeo de 10 mca y requiere menor caudales de alimentación de 19.09, 
20.43 y $17.911 /$ min a presiones máximas de trabajo de 30, 35 y 38 mca según longitud de tubería. En la Fig. 15, y las Tabla 4, para carrera de válvula $S=6 \mathrm{~mm}$, se puede observar que la bomba de ariete evaluada con longitudes de tuberías de $\mathrm{L}=1, \mathrm{~L}=2$ y $\mathrm{L}=3 \mathrm{~m}$, requiere respectivamente mayor caudal de alimentación en 43.67, 42.34 y $42.13 \mathrm{l} / \mathrm{min}$ obtenidas en alturas de bombeo de 10 mca y requiere menor caudales de alimentación de 26.52 , 29.33 y $21.581 / \mathrm{min}$ a presiones máximas de trabajo de 30, 46 y 50 mca según longitud de tubería.

En la Figura 16, y las Tabla 5, para carrera de válvula $S=9$ $\mathrm{mm}$, se puede observar que la bomba de ariete evaluada con longitudes de tuberías de $\mathrm{L}=1, \mathrm{~L}=2$ y $\mathrm{L}=3 \mathrm{~m}$, requiere respectivamente mayor caudal de alimentación en 43.79, 47.53 y 44.50 . 1/min obtenidas en alturas de bombeo de 10 mca y requiere menor caudales de alimentación de 27.01, 27.09 y $23.53 \mathrm{l} / \mathrm{min}$ a presiones máximas de trabajo de 30, 46 y 52 mca según longitud de tubería. Finalmente, según las gráficas de las Figuras. 14, 15 y 16, y las Tablas 3, 4 y 5 los menores caudales de alimentación en diferentes carrera de válvula de impulso con $S=3, S=6$ y $\mathrm{S}=9 \mathrm{~mm}$, fueron dadas por la tubería de mayor longitud $\mathrm{L}=3 \mathrm{~m}$, siendo estas $17.91,21.58$ y $23.53 \mathrm{l} / \mathrm{min}$ dado en alturas máximas de trabajo con descarga de bombeo nulo, por otro lado los caudales mayores de alimentación en la tubería de $3 \mathrm{~m}$ de longitud, ocurre en las alturas de bombeo de $10 \mathrm{~m}$ y tienen los valores de $35.08,42.13$ y $44.50 \mathrm{l} / \mathrm{min}$ de acuerdo a la carrera de válvula y estos caudales son menores a las de las otras tuberías.

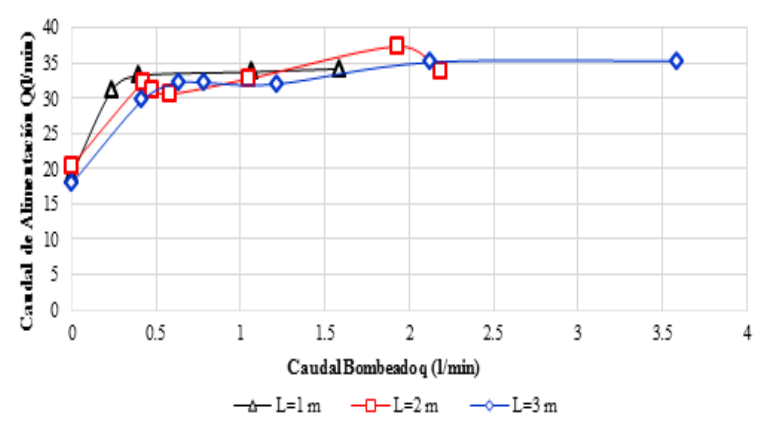

Figura 14.- Caudal de Bombeo rs. Caudal de Alimentación de BAH 1.1/2 Para longitudes de tuberias de prueba, con Carrera $\mathrm{S}=\mathbf{3} \mathrm{mm}$

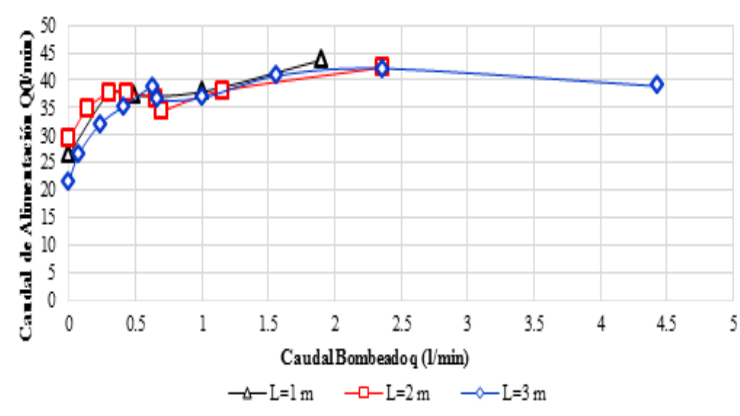

Figura 15.- Caudal de Bombeo rs. Caudal de Alimentación de BAH 1.1/2 Para longitudes de tuberias de prueba, con Carrera $\mathrm{S}=6 \mathrm{~mm}$

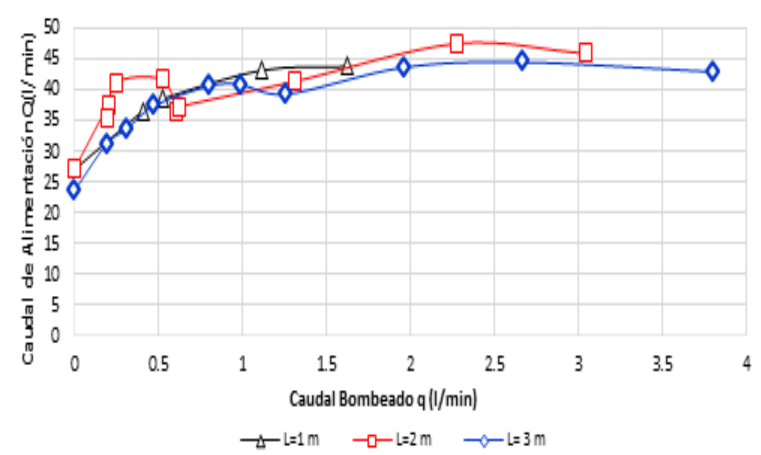

Figura 16.- Caudal de Bombeo rs. Caudal de Alimentación de BAH 1.1/2 Para longitudes de tuberias de prueba, con Carrera $\mathrm{S}=9 \mathrm{~mm}$

Finalmente evaluando los resultados en las gráficas de las Figuras. Del 8 al 13 y Tablas 3,4 y 5 en lo correspondiente a la tubería de $3 \mathrm{~m}$ de longitud, con carreras de la válvula de impulso de 3, 6 y $9 \mathrm{~mm}$, se puede observar claramente que puede operar dentro de los rangos de eficiencia 33\% al $66 \%$ recomendado a partir de los $10 \mathrm{~m}$ de altura de bombeo, consiguiendo mejores eficiencias en 20, 25 y 30 $\mathrm{m}$ de alturas. Operando en $20 \mathrm{~m}$ de altura a eficiencias de $48.87,54.63$ y $64.16 \%$ y caudales de bombeo de $0.79,1.01$ y $1.26 \mathrm{l} / \mathrm{min}$ y volúmenes por día 1137.6, 1454.40 y 1814.40 litros. También es posible operar en $25 \mathrm{~m}$ de alturas con eficiencias de 49.20, 46.65 y $60.34 \%$ y caudales de bombeo de $0.63,0.67$ y $0.99 \mathrm{l} /$ min consiguiendo volúmenes por día de 907.20, 964.80 y 1425.60 litros. Finalmente se puede también obtener $30 \mathrm{~m}$ de altura consiguiendo eficiencias de 42.31, 48.96 y $59.11 \%$ y caudales de $0.42,0.61$ y $0.801 / \mathrm{min}$ con volúmenes por día de 604.80, 907.2 y 1152 litros, siendo todos estos puntos muy adecuados.

\section{Conclusiones}

Las máximas alturas de bombeo alcanzadas por la bomba de ariete hidráulico BAH 1.1/2, en las carrera de 3, 6 y $9 \mathrm{~mm}$ de la válvula de impulso, se logró en la tubería de $3 \mathrm{~m}$ de longitud, Siendo esta altura de 38, 50 y $52 \mathrm{~m}$ con caudal de bombeo nulo y descargas elevadas de 2.13, 2.36 y $2.67 \mathrm{l} / \mathrm{min}$ a $10 \mathrm{~m}$ de altura de descarga.

Las mayores eficiencias alcanzadas por la bomba de ariete hidráulico BAH 1.1/2 en las carrera 3, 6 y 9 mm de la válvula de impulso, fueron conseguidas por la tubería de $3 \mathrm{~m}$ longitud, siendo estas eficiencias de 60.63, 55.93 y $59.92 \%$ con caudales de bombeo de $2.13,2.36$ y 2.67 1/ min a $10 \mathrm{~m}$ de altura de descarga.

Los menores caudales de alimentación requeridos en carrera de 3, 6 y $9 \mathrm{~mm}$ de la válvula de impulso, fueron dadas por la tubería de $3 \mathrm{~m}$ longitud, siendo estas 17.91, 21.58 y $23.53 \mathrm{l} / \mathrm{min}$ conseguidos en alturas máximas de bombeo con caudales de bombeo nulo, por otro lado los caudales mayores de alimentación en la tubería de $3 \mathrm{~m}$ de longitud, en las respectiva carreras, ocurre en las alturas de bombeo de $10 \mathrm{~m}$ siendo los valores de 35.08, 42.13 
y $44.50 \mathrm{l} / \mathrm{min}$ siendo estos valores menores a las de las otras tuberías evaluada. La bomba de ariete BAH 1.1/2 operó eficientemente con tubería de $3 \mathrm{~m}$ de longitud y con carreras de la válvula de impulso de $9 \mathrm{~mm}$, con lo cual se logra alcanzar con facilidad respectivamente alturas de bombeo de 20, 25 y $30 \mathrm{~m}$ con eficiencia $64.16,60.34$ y $59.11 \%$ a caudales bombeados de $1.26,0.99$ y $0.801 / \mathrm{min}$, consiguiendo de este modo bombear un volumen por día de $1814.40,1425.60$ y 1152 litros, trabajando dentro de los rangos de $33 \%$ al $66 \%$ eficiencia recomendado por los fabricantes y las bibliografía revisadas.

\section{Recomendaciones}

Se recomienda probar este modelo BAH 1.1/2 con mayores longitudes de tubería de alimentación y de otros materiales como fierro galvanizado (FG), polietileno (PE) y en las mismas condiciones en las que fue evaluado con el fin de obtener nuevas eficiencias.

\section{Literatura citada}

Ortiz, V. 1993. Optimización del Ariete Hidráulico: Modelo Matemático y Físico. Lima, La Molina. Tesis Mg Sc. Escuela de Post-Grado, UNALM. 116 p.

Rodríguez Díaz, H. 2002. Hidráulica Experimental. Colombia Mayo 2002. Edit. ECI. 337 p.

Saldarriaga V. J. G. 1998. Hidráulica de Tuberías. Bogotá. Mc Graw Hill Interamericana S.A. 564p.

Streeter, V. 2011. Mecánica de Fluidos. Trad. The McGraw-Hill. 9 ed. Editorial McGraw-Hill Interamericana S.A. 740p. 